\title{
Genetic-Optimized Pulse Coding Technique for Brillouin Distributed Optical Fiber Sensing
}

\author{
Xizi Sun ${ }^{1}$, Zhisheng Yang,", Xiaobin Hong,", Simon Zaslawski ${ }^{2}$, Sheng Wang ${ }^{1}$, Marcelo A. Soto ${ }^{3}$, Xia Gao ${ }^{1}$, \\ Jian $\mathrm{Wu}^{1}$ and Luc Thévenaz ${ }^{2}$ \\ ${ }^{I}$ State Key Laboratory of Information Photonics \& Optical Communications, Beijing University of Posts and Telecommunications, Beijing \\ 100876, China \\ ${ }^{2}$ EPFL Swiss Federal Institute of Technology, Institute of Electrical Engineering, SCI STI LT, Station 11, CH-1015 Lausanne, Switzerland \\ ${ }^{3}$ Department of Electronics Engineering, Universidad Técnica Federico Santa María, Valparaíso, Chile \\ Corresponding author: zhisheng.yang@epfl.ch; xbhong@bupt.edu.cn
}

\begin{abstract}
A single code sequence designed by a genetic searching algorithm is proposed to enhance the signal-to-noise ratio of Brillouin distributed sensors without hardware modification, enabling high-performance and cost-effective sensing over a 200-km-loop setup. @ 2021 The Author(s)
\end{abstract}

\section{Introduction}

Over the past three decades, time-domain distributed optical fiber sensors (DOFS) have experienced a substantial development, demonstrating that the overall performance of DOFS can be rigorously quantified by the measurement signal-to-noise ratio (SNR) [1]. Whilst single-pulse based configurations have reached their ultimate performance, the use of optical pulse coding, based on launching one or several sequences of pulses into the sensing fiber, is widely considered as an efficient method to further enhance the SNR [2]. However, it turns out that conventional code types such as Golay, Simplex and cyclic codes present practical limitations [1,3-5], such as the need of extra measurement/decoding time [1,5], compromised SNR enhancement [1] or sensitivity to the sequence amplitude nonuniformity [3,4]. To overcome these limitations, a novel coding technique based on deconvolution has been recently proposed [5], offering the adaptability to non-uniform sequence, one-sequence encoding and instantaneous decoding. In this technique, a pseudo-random binary sequence (PRBS) assisted by a constrained least-square (CLS) filter is used, demonstrating an attractive SNR improvement (so-called coding gain) in specific code lengths, whilst using the same hardware and measurement time as a standard single-pulse configuration. However, the coding gain of the PRBS approach for any code length has not been rigorously quantified yet, and it still remains unclear if the maximum possible coding gain has been reached for deconvolution-based coding technique.

In this paper, an analytical expression of the coding gain for the deconvolution-based coding approach is built, enabling to evaluate the performance of any kind of code sequence. Based on this, the previously proposed PRBS code combined with CLS filter is firstly assessed, finding that its coding gain underperforms that of conventional codes, especially when using long code lengths. To further enhance the SNR, a new type of unipolar code sequence is here designed using a dedicatedly-developed distributed genetic algorithm (DGA). The presented analysis and experimental results demonstrate that the coding gain offered by this novel code sequence (here designed as generically-optimized code, in short GO-code) is comparable to that of conventional codes, whilst requiring only one code sequence and keeping all advantages of the deconvolution concept. The capability of the proposed GO-code is here experimentally demonstrated using the most standard implementation of a distributed sensors based on Brillouin optical time-domain analysis (BOTDA).

\section{Principle}

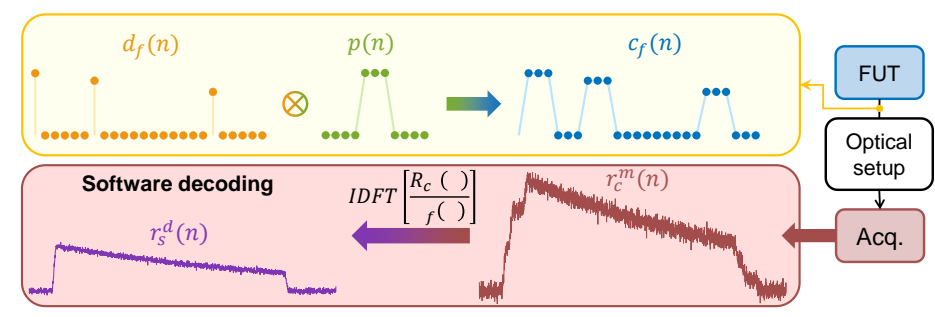

Fig. 1. Principle of the decoding process based on the concept of deconvolution.

For any linear time-invariant DOFS, the acquired coded fiber response, designated here as $r_{c}^{m}(n)$, can be commonly expressed by the linear convolution between the coded optical pulse sequence $c_{f}(n)$ and the fiber impulse response $h(n)$, merged with a zero-mean additive noise $e(n)$, as $r_{c}^{m}(n)=c_{f}(n) \otimes h(n)+e(n)$. The integer $n$ is linearly related to the time $t$ through the sampling rate $f_{s}$, as $t=n / f_{s}$, and the sign $\otimes$ denotes linear convolution. Note 
that the optical pulse sequence $c_{f}(n)$ launched into the sensing fiber may exhibit a non-uniform amplitude envelop due to the gain saturation of the optical amplifier (e.g. an erbium-doped fiber amplifier - EDFA) used to boost the optical power of the code sequence, and can be further expressed as the linear convolution between a single-pulse signal $p(n)$ and a series of delta function $d_{f}(n)$, as $c_{f}(n)=d_{f}(n) \otimes p(n)$ (see Fig. 1). This way the coded fiber response $r_{c}^{m}(n)$ and its discrete frequency-domain representation can be expressed as:

$$
r_{c}^{m}(n)=d_{f}(n) \otimes p(n) \otimes h(n)+e(n) \stackrel{F}{\rightarrow} R_{c}^{M}(k)=D_{F}(k) P(k) H(k)+E(k)
$$

where $F$ denotes discrete Fourier transform (DFT) operation. Since $d_{f}(n)$ can be obtained by measuring the energy of each optical pulse in $c_{f}(n)$ at the fiber input, the targeted single-pulse response can be retrieved by performing the following decoding process based on the known decoding function $D_{F}(k)$ [5]:

$$
r_{s}^{d}(n)=\operatorname{IDFT}\left[\frac{R_{c}^{M}(k)}{D_{F}(k)}\right]=\operatorname{IDFT}\left[P(k) H(k)+\frac{E(k)}{D_{F}(k)}\right]=r_{s}(n)+\operatorname{IDFT}\left[\frac{E(k)}{D_{F}(k)}\right]
$$

as shown in Fig. 1, where $r_{S}^{d}(n)$ is the decoded single-pulse response that contains the targeted single-pulse response $r_{s}(n)=p(n) \otimes h(n)$ and a noise term affected by $D_{F}(k)$. This determines the coding gain $G_{C}$ of the used code, expressed as the ratio of the noise standard deviation before $\left(\sigma_{e}\right)$ and after decoding $\left(\sigma_{d e}\right)$ :

$$
G_{C}=\frac{\sigma_{e}}{\sigma_{d e}}=\frac{1}{\sqrt{\left\langle\left|1 / D_{F}(k)\right|^{2}\right\rangle}}
$$

It appears evident that to maximize the coding gain $G_{C}$, it is required to dedicatedly design $d_{f}(n)$, which should make $D_{F}(k)$ as flat as possible according to the Cauchy inequality. An alternative is to use a PRBS code [5] due to its random distribution of 0's and 1's elements within the sequence; however, the coding gain provided by PRBS code is not ideal as pointed out by the green curve in Fig. 2(a). The coding gain is evaluated based on Eq. (3), as a function of the pulse energy enhancement factor $F_{E}=\sum d_{f}(n)$, which represents the increment of energy launched into the sensing fiber compared to the single-pulse energy. In the figure, the standard reference coding gain $G_{r}=\sqrt{F_{E} / 2}$ offered by conventional coded-DOFS (e.g. Simplex and Golay codes $[1,4]$ ) is provided (blue curve) for comparison. Results indicate that the coding gain of PRBS is much lower than conventional codes for most code lengths. By applying a CLS filter (i.e., using the decoding function expressed as $1 /\left(D_{F}(k)+\alpha|P(k)| /\left|D_{F}(k)\right|\right)$ [5], denoted as $1 / D_{C L S}(k)$, where $\alpha$ is a filtering coefficient and $|P(k)|$ is a high-pass filter), the performance of the PRBS code can be enhanced [5], with the penalty of signal distortions that can be made negligible, depending on the value of $\alpha$. Nevertheless, it turns out that the PRBS code combined with an optimized CLS filter (with an optimized $\alpha=20$ as will be demonstrated in the next section) still results in a coding gain (black curve in Fig. 2) lower than that of conventional codes $G_{r}$, especially for long code lengths. Therefore, a new type of $d_{f}(n)$ that is dedicatedly designed to maximize the coding gain $G_{C}$ expressed by Eq. (3) is still desirable.
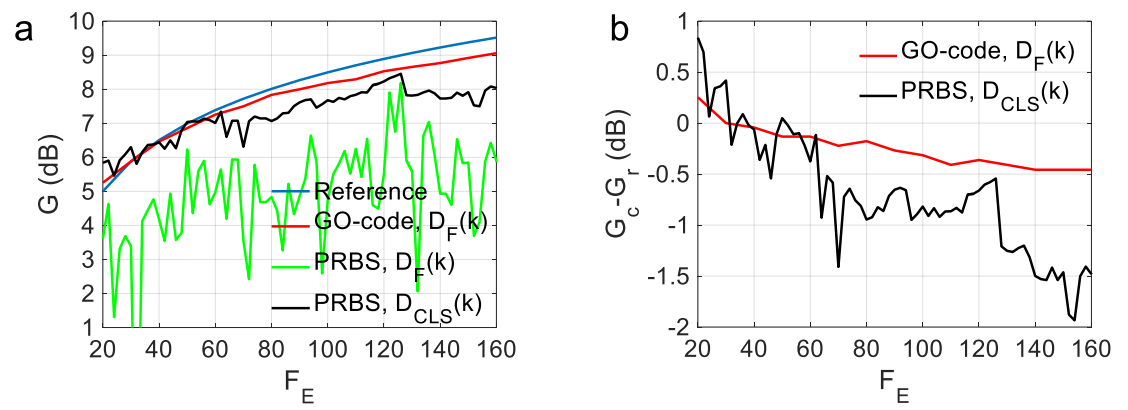

Fig. 2. (a) $G_{C}$ of the proposed GO-code, PRBS code and PRBS with $D_{C L S}(k)$, as a function of $F_{E}$, compared to standard reference coding gain $G_{r}$; (b) SNR difference between $G_{C}$ and $G_{r}$, for the proposed GO-code and the PRBS code combined with $D_{C L S}(k)$.

To the best of our knowledge it is however extremely complex to analytically find the optimum code sequence that maximizes $G_{C}$, so that a distributed genetic algorithm (DGA) based on the principle of natural genetics evolution [6] is here delicately developed to efficiently search for the optimal code (here designated as GO-code). The performance of the proposed GO-code is illustrated by the red curve in Fig. 2(a), outperforming PRBS coded combined with CLS filter. Although the obtained SNR still underperforms that provided by conventional codes, the maximum difference in all analyzed cases is $<0.5 \mathrm{~dB}$ (see red curve in Fig.2 (b)), which can be considered negligible in most applications.

\section{Experimental results and discussion}




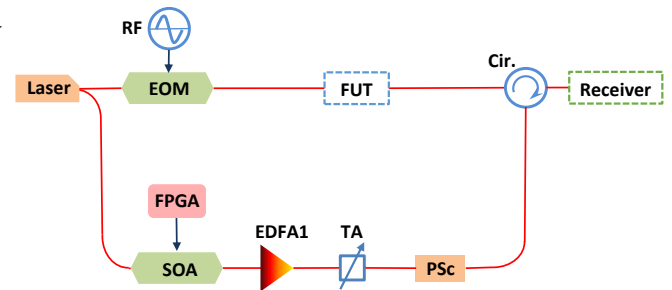

$\mathrm{b}$

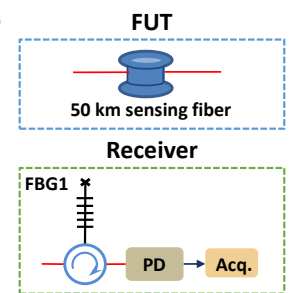

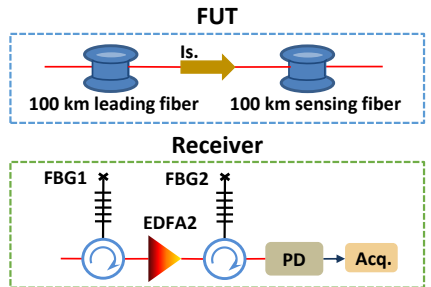

Fig. 3. (a) Experimental setup; Fiber under test (FUT) and receiver part of (b) the 50-km-long sensing fiber configuration and (c) the 200-km-long fiber loop configuration.

The performance of the PRBS code and the proposed GO-code is investigated and compared based on a standard BOTDA setup, as shown in Fig. 3(a). In such a setup, the use of a single pulse (for reference), PRBS code and GOcode can be readily switched by solely alternating the pulse/coding digital signal uploaded to the FPGA.
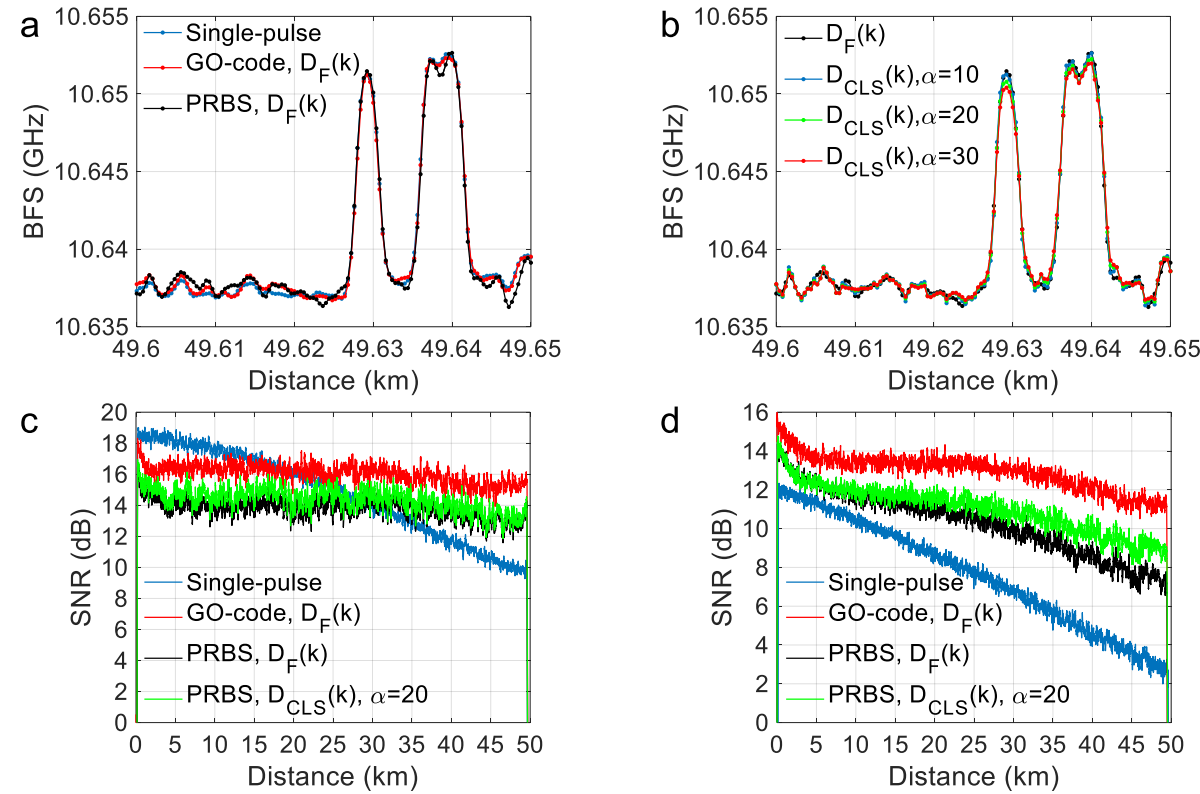

Fig. 4 (a)-(c) Experimental results in the case of 20 ns pulse: (a) the hotspot BFS when using decoder $D_{F}(k)$; (b) the hotspot BFS for PRBS when using decoder $D_{C L S}(k)$; (c) SNR of the decoded response; (d) SNR of the decoded response for $10 \mathrm{~ns}$ pulse. The blue curve in Fig. 4(a) is obtained with 16384 averages, all rest curves are obtained with 1024 averages.

For the sake of visual clarity, comparisons are firstly carried out over a 50-km-long sensing fiber with two hotspots of $2 \mathrm{~m}$ and $5 \mathrm{~m}$ (separated by a $5 \mathrm{~m}$ unperturbed section) at the fiber far-end. In the receiver, the high-frequency probe sideband is directly detected by a $125 \mathrm{MHz}$ photodetector (PD) and a real-time data acquisition system with a sampling rate of $250 \mathrm{MS} / \mathrm{s}$, as illustrated in Fig. 3(b). The single-pulse duration is set to $20 \mathrm{~ns}$ and the peak power is optimized to $23 \mathrm{dBm}$, providing an optimized single-pulse Brillouin gain of $2.5 \%$. The energy enhancement factor $F_{E}$ for both PRBS code and GO code are set as $40(=100 \% / 2.5 \%)$ to avoid possible decoding distortion [4]. The corresponding $d_{f}(n)$ for both codes are precisely retrieved based on the actual envelop of the coding sequences detected at the output of EDFA1. After performing the decoding process expressed by Eq. (2), single-pulse responses for both codes are retrieved, respectively. Then, the distributed BFS profiles corresponding to both cases are estimated using crosscorrelation method [7] and are shown by red and black curves in Fig. 4(a). By comparing with a reference curve (black) obtained from single-pulse measurement with 16384 averages (for a high SNR and such large averages is only performed for this curve), it can be found that there is no signal impairment for both codes, demonstrating the correctness of Eq. (2). Meanwhile, as shown in Fig. 4(c), the SNR of the proposed GO-code (red) outperforms the PRBS code (black) by $2.5 \mathrm{~dB}$, consistent with the results shown in Fig. 2(a). Then, CLS filters with $\alpha=10,20$ and 30, respectively, are applied to the PRBS-coded response, aiming to improve the performance of PRBS code and to optimize filter parameters. The obtained results are shown in Fig. 4(b), in which larger BFS errors can be found with larger value of $\alpha$, i.e., $0.6 \mathrm{MHz}$ error for $\alpha=20$ and $1 \mathrm{MHz}$ for $\alpha=30$. Considering $\alpha=20$ is a good condition that simultaneously minimizes the BFS error and maximizes the SNR improvement, the SNR profile of the decoded response in such case is shown by the green curve in Fig. 4(c). Although the obtained SNR is higher than the case decoded by Eq. (2) with PRBS code, it is still poorer than the use of GO-code. Note that this seems in contrast with 
the theory indicated in Fig. 2, where the PRBS code with the CLS filter shows comparable performance to that of GOcode. This is mainly because all the coded sequences evaluated by Fig. 2 are assumed to be flat, while the practical sequences in experiments are uneven due to the EDFA transient effects, so that the spectral distribution of the coded sequence is changed and this disturbance has an uncertain impact on these non-optimized codes. To further demonstrate the performance difference between these two codes in the case of long code lengths, a comparison with $1 \mathrm{~m}$ spatial resolution is performed, which allows us to use a larger $F_{E}(=200)$. The SNR profiles are shown in Fig. 4(d), demonstrating that the GO-code substantially outperforms PRBS code in the case of longer code lengths.

Table 1. Experimental parameters in the GO-coded BOTDA when using 200-km-long fiber loop.

\begin{tabular}{|c|c|c|c|c|c|c|c|}
\hline $\begin{array}{c}\text { Spatial } \\
\text { resolution } \\
(\mathrm{m})\end{array}$ & $F_{E}$ & $\begin{array}{c}G_{c} \\
(\mathrm{~dB})\end{array}$ & $\begin{array}{c}\text { Scanning } \\
\text { range } \\
(\mathrm{MHz})\end{array}$ & $\begin{array}{c}\text { Scanning } \\
\text { step } \\
(\mathrm{MHz})\end{array}$ & $\begin{array}{c}\text { Average } \\
\text { times }\end{array}$ & $\begin{array}{c}\text { Measurement } \\
\text { time } \\
(\text { minute })\end{array}$ & $\begin{array}{c}\text { Decoding } \\
\text { time } \\
(\text { second })\end{array}$ \\
\hline 2 & 40 & 6.2 & 150 & 1 & 1024 & 2.8 & 1.8 \\
\hline 1 & 200 & 9.3 & 300 & 1 & 1024 & 5.7 & 3.6 \\
\hline
\end{tabular}

To demonstrate the ultimate performance of the proposed GO-code, experiments are conducted over a sensing range of $100 \mathrm{~km}$ in a $200 \mathrm{~km}$-long fiber loop, with a pre-amplification configuration as shown in Fig. 3(c). Measurements with $2 \mathrm{~m}$ and $1 \mathrm{~m}$ spatial resolutions are carried out and compared to optimized single-pulse schemes with same averages, based on parameters shown in Table 1. As shown in Fig. 5, the BFS uncertainties at the fiber far end obtained by the GO-code scheme are reduced by factor 4.2 and 8.59 with respect to those obtained by the singlepulse scheme, matching perfectly the theoretically predicted coding gain of $6.2 \mathrm{~dB}$ and $9.3 \mathrm{~dB}$, respectively.
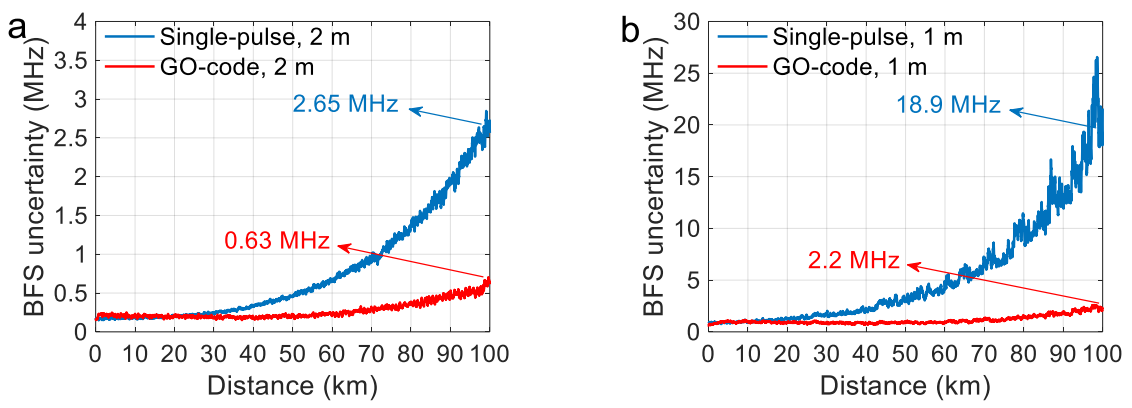

Fig. 5. BFS uncertainty along the sensing fiber for (a) $2 \mathrm{~m}$ and (b) $1 \mathrm{~m}$ spatial resolution.

\section{Conclusion}

In this work, a single-sequence genetic-optimized code for enhancing the SNR of Brillouin distributed sensing has been proposed. Experimental results on a standard BOTDA system have demonstrated the capabilities of the proposed code to achieve all conceptual advantages of the pulse coding method, leading to results outperforming PRBS coding methods relying on the CLS filtering and obtaining a similar coding gain compared to conventional codes. In addition, the proposed code requires no hardware modifications on a single-pulse DOFS, enabling an SNR enhancement with no penalty on the measurement time. The verified performance enhancement is achieved by only modifying the software in relation to the pulse generation and post-processing (decoding), thus offering a simple upgrade towards higher performance for distributed sensing. The presented approach can benefit not only BOTDA as demonstrated here, but also any linear time invariant system requiring the retrieval of the single-pulse response with a high SNR.

Acknowledgements: National Natural Science Foundation of China under Grant 61875018. M. A. Soto acknowledges AC3E Basal Project FB0008.

\section{References}

[1] A. H. Hartog, An Introduction to Distributed Optical Fiber Sensors (Boca Raton, 2017).

[2] M. A. Soto and L. Thévenaz, "Modeling and evaluating the performance of Brillouin distributed optical fiber sensors," Opt. Express 21, 31347-31366 (2013).

[3] M. A. Soto, G. Bolognini, F. D. Pasquale, and L. Thévenaz, "Long-range Brillouin optical time-domain analysis sensor employing pulse coding techniques," Meas. Sci. Technol. 21, 094024 (2010).

[4] Z. Yang, Z. Li, S. Zaslawski, L. Thévenaz, and M. A. Soto, "Design rules for optimizing unipolar coded Brillouin optical time-domain analyzers," Opt. Express 26, 16505-16523 (2018).

[5] X. Sun, Z. Yang, X. Hong, S. Zaslawski, S. Wang, J. Wu, and L. Thévenaz, "Boosting the performance of distributed optical fiber sensors based on adaptive decoder," in 26th International Conference on Optical Fiber Sensors (2018), paper ThE102.

[6] M. Tomassini, "A survey of genetic algorithms, " Annual Reviews of Computational Physics III, pp. 87-118 (1995).

[7] S. M Haneef, Z. Yang, L. Thévenaz, D. Venkitesh, and B. Srinivasan, "Performance analysis of frequency shift estimation techniques in Brillouin distributed fiber sensors," Opt. Express 26, 14661-14677 (2018). 